\title{
La légitimité des transferts de pratiques de management au sein de la firme « locale-globale »
}

\section{Claire Burlat}

\section{OpenEdition}

\section{Journals}

Édition électronique

URL : http://journals.openedition.org/communicationorganisation/926

DOI : 10.4000/communicationorganisation.926

ISSN : 1775-3546

Éditeur

Presses universitaires de Bordeaux

Édition imprimée

Date de publication : 1 décembre 2009

Pagination : $70-81$

ISBN : 978-2-86781-719-9

ISSN : $1168-5549$

Référence électronique

Claire Burlat, "La légitimité des transferts de pratiques de management au sein de la firme "localeglobale » », Communication et organisation [En ligne], 36| 2009, mis en ligne le 11 mars 2011, consulté le 19 avril 2019. URL : http://journals.openedition.org/communicationorganisation/926 ; DOI :

10.4000/communicationorganisation.926 
Dossier : Pour une approche communicationnelle de l'individu au travail

\begin{abstract}
Résumé
Les firmes multinationales cherchent souvent à faire adopter par leurs filiales à l'étranger le modèle de gestion des individus de la maisonmère. Lorsque les transferts de pratiques managériales correspondent à une stratégie économique et sociale unifiée, au-delà de la recherche d'effets d'image, les règles de l'organisation sont modifiées. La mise en œuvre d'un changement profond qui altère les rapports de l'individu au travail va rarement de soi. L'étude de trois cas montre que de tels changements deviennent légitimes par la négociation sociale. La variété des processus de légitimation témoigne de l'ancrage culturel des trois filiales. À chaque fois, ils supposent une transformation des identités professionnelles et des modes de socialisation.
\end{abstract}

Mots clés

Management, légitimation, négociation sociale, firme locale-globale

Abstract

Multinational companies tend to transfer to subsidiaries management practices they have developed in the mother company. When the aim of such operations not only relies on image stakes but also on business model strategies, the organizational rules of subsidiaries are modified. The implementation of such a change is a challenge since it has an important impact on the relationships employees develop with their occupation. Three case studies show that these changes gain legitimacy through industrial relationship. Different ways of legitimating account for the cultural embedment of subsidiaries. In each case, the legitimacy of these changes relies on the transformation of employee socialisation and identity in the workplace.

\title{
Keywords
}

Management, legitimisation, industrial relationship, local-global company

Claire Burlat est doctorante en sociologie au laboratoire MODYS, Mondes et Dynamiques des Sociétés, de l'Université Lyon II et Attachée Temporaire à l'Enseignement et à la Recherche (ATER) au CELSA, École des Hautes Etudes de l'Information et la Communication de l'Université Paris-Sorbonne. 
La légitimité des transferts de pratiques de management...

\section{La légitimité des transferts de pratiques de management au sein de la firme « locale-globale » Claire Burlat}

claire.burlat@ celsa.paris-sorbonne.fr

Beaucoup de discours sur la mondialisation des entreprises réduisent ce phénomène à une division internationale du travail binaire où la création de valeur serait le fait des maisons-mères alors que les filiales seraient condamnées à produire au moindre coût (Berger, 2006). Cependant, les filiales peuvent aussi produire de la valeur, notamment lorsque la maison-mère adopte une logique d'investissement dans leur gestion des ressources humaines (Mendez, 1994). Aussi, les firmes multinationales cherchent-elles souvent à faire adopter par leurs entités à l'étranger un modèle unifié de gestion des hommes. Les conditions de la mise en œuvre optimale de ces pratiques de management dans les filiales sont largement traitées par la littérature gestionnaire (Kogut, Zander, 1993 ; Davenport et al., 1998 ; Kostova, Roth, 2002). Nous proposons alternativement de concentrer l'attention sur les nouveaux rapports au travail induits par ces transferts et des processus qui en assurent la légitimité. Il s'agit donc d'emprunter un objet d'étude propre aux sciences de gestion (les transferts de pratiques de management) pour comprendre un phénomène social du point de vue des processus communicationnels (Habermas, 1987) et organisationnels qui le structurent. Cet article montre la diversité de tels processus au travers de l'analyse comparée de trois situations de transferts de pratiques managériales au sein d'un même groupe international de services. Il met en évidence les formes très différentes prises par la négociation sociale et leur influence sur la transformation des modes de socialisation professionnelle.

\section{Les transferts de pratiques de management : quelle légitimité ?}

Si certains transferts de pratiques de management peuvent se réduire à des opérations «coquilles vides » qui correspondent à la recherche d'un simple effet d'image, la plupart relèvent de la volonté de mettre en œuvre une stratégie économique et sociale unifiée. BSN (devenu le groupe Danone en 1994) fait ici figure de cas d'école: le groupe déploie dans les filiales des principes de gestion des hommes conformes au «double projet économique et social» initié dès les années 1970 par son emblématique PDG Antoine Riboud. Les changements ne sauraient se limiter à une intégration artificielle par les acteurs locaux des pratiques transférées: ils visent à modifier 
Dossier : Pour une approche communicationnelle de l'individu au travail

largement les règles de l'organisation. Or, la mise en œuvre d'un changement profond qui altère les rapports de l'individu au travail (Sainsaulieu, 1990) va rarement de soi. Ainsi, dans le cas d'une fusion-acquisition, un changement orchestré par la direction peut produire des effets négatifs sur la qualité du travail et sur l'emploi s'il n'apparaît pas légitime de prime abord aux salariés (Bernoux, Burlat, 2007). Si le transfert prend la forme d'une domination de la maisonmère qui tente d'imposer son système de management à la filiale, il peut être rejeté socialement. Les rapports de domination ne sont légitimes que dans des cas bien précis ${ }^{29}$ (Weber, 1971) qui se présentent rarement dans le contexte d'une firme multinationale. La mise en place du modèle de management de la maison-mère sous la forme d'une « régulation de contrôle » (Reynaud, 1997) où seule la volonté de la maison-mère guiderait les changements, présente donc peu de gage de réussite. Dès lors, de quelle manière le transfert d'un modèle de management peut-il finalement être légitimé au sein d'une filiale d'une firme multinationale? Quelles en sont les modalités communicationnelles?

Les processus de légitimation de la mise en place de nouvelles pratiques de gestion peuvent prendre d'autres voies. Tout d'abord, la mise en œuvre des changements peut être confiée aux acteurs de terrain. Cependant ces changements « spontanés » conduisent souvent à la reproduction des anciennes pratiques professionnelles (Guilbert, 2008). Dans ce cas, le changement est limité par le phénomène de la «régulation autonome » (Reynaud, 1997). Il peut aussi prendre la forme d'une « régulation conjointe » (Reynaud, 1997) où les règles de la maison-mère ne sont ni imposées, ni délaissées au profit des pratiques informelles, mais discutées. Soumis à la négociation sociale, les changements font alors l'objet d'un débat institutionnel entre les concepteurs de l'organisation formelle ou leurs représentants et les acteurs dont le travail est affecté.

Dans cette perspective, les relations sont alors structurées par deux logiques : une «activité communicationnelle » animée par une volonté d'intercompréhension et d'accord (Habermas, 1987) et une activité stratégique relative à un calcul de l'acteur (Crozier, Friedberg, 1977). Sous la contrainte de la nécessité de l'accord, la mise en oeuvre de

29 Il s'agit des cas de la légitimité traditionnelle fondée sur le caractère sacré de la tradition, de la légitimité charismatique s'appuyant sur des figures héroïques et de la légitimité rationnelle-légale fondée sur la légalité des titres de ceux qui exercent une domination. 
nouvelles règles devient l'occasion d'un vivre ensemble (Thuderoz, Giraud-Héraud, 2000) au cours duquel les rapports sociaux traditionnels s'éprouvent et se transforment. Dans ce cas, la négociation vient perturber «les identités usuelles [et] c'est toute la légitimité de l'ancien compromis social qui est atteinte » (Thuderoz, Giraud-Héraud, $2000: 21$ ).

L'étude du cas de l'internationalisation d'une entreprise multinationale d'origine française spécialisée dans les services publics urbains permet d'éclairer empiriquement ce débat ${ }^{30}$. Cette firme de type «locale-globale» (Lorrain, 2005 ; Brochier et al, 2007) illustre avec pertinence le cas d'une entreprise qui cherche à faire adopter à ses filiales un même modèle de management tout en l'adaptant à leurs spécificités économiques et sociales. L' «activité communicationnelle » observée (la négociation sociale) correspond à une logique de «régulation conjointe » où le comportement stratégique d'un acteur (la maison-mère) vise à transférer durablement ses propres règles (le modèle de management) à des acteurs locaux (la filiale). La variété des processus de légitimation dépend alors du résultat de la confrontation de ces deux stratégies d'acteurs. L'introduction négociée d'un nouveau type de management dans trois implantations européennes de cette firme (en France, en Suède et en Roumanie) constitue la base de l'analyse. Le modèle transféré est celui du «management par les compétences » (Zarifian, 2004) reposant sur des principes de décentralisation des organisations de travail, d'autonomie et de responsabilisation des salariés à l'égard des résultats (Reynaud, 2001). Dans ce modèle, le développement de l'employabilité par l'organisation constitue la contrepartie à l'appel à la performance et la responsabilité accrue des salariés. Il implique dans chaque filiale un nouveau «trade-off» (Reynaud, 2001: 17), à savoir, la redéfinition du compromis social local antérieur, afin d'aboutir à un meilleur positionnement stratégique de l'entreprise en faveur de la qualité de service.

30 Cette recherche a été menée dans le cadre d'une thèse sous contrat CIFRE de 2005 à 2008. La méthodologie était constituée d'entretiens semi-directifs (environ quarante dans chaque filiale) auprès de managers expatriés et locaux, de praticiens en gestion des ressources humaines et de responsables syndicaux, ainsi que des observations participantes (trois mois par filiale) et enfin l'exploitation de documents internes par des analyses de contenu. 
Dossier : Pour une approche communicationnelle de l'individu au travail

Cas $n^{\circ} 1$ : substituer une identité « de compétence » à une identité fusionnelle en France

La filiale française exploite le réseau de transport collectif d'une communauté urbaine de près de 700000 habitants. Le réseau est dit multimodal car il inclut du transport par bus et plus récemment par tramway. Le développement des lignes de tramway engendre la restructuration du réseau de bus et la diminution de ce mode de transport. L'entreprise emploie 2000 personnes. Parmi les 1300 conducteurs, 300 sont affectés au tramway. L'introduction des pratiques de management de la maison-mère est caractérisée par le déploiement d'un accord-cadre concernant «les principes du développement des compétences et de la progression professionnelle ». La négociation doit porter principalement sur les modalités de l'intégration des nouveaux salariés, le rôle de la fonction tutorale dans la transmission de savoir-faire, le développement de parcours professionnels et la mise en place d'une gestion prévisionnelle des emplois et des compétences. Bien qu'un accord d'entreprise ait finalement été signé avec les délégués syndicaux, cette négociation s'avère particulièrement conflictuelle. Elle institutionnalise de nouvelles formes de socialisation et d'identités naissantes en concurrence avec les anciennes. À l'époque où elle exploitait uniquement le réseau de bus avant l'arrivée du tramway, l'entreprise se caractérisait par la «paix sociale». L'organisation du travail reposait sur le principe d'affectation «à vie » des conducteurs sur une seule ligne. La gestion des individus était conditionnée par les principes de l'ancienneté et l'égalité de traitement. Les conducteurs trouvaient dans le collectif une ressource majeure fondatrice de leur identité professionnelle de type «fusionnelle » (Sainsaulieu, 1977). Ils constituaient un groupe solidaire et homogène duquel étaient exclus les nouveaux conducteurs le temps de leur phase d'intégration.

La stabilité de ce système est remise en cause par l'introduction du tramway dans l'organisation. Les tracés et horaires du réseau changent constamment et les nouvelles technologies nécessitent désormais adaptation, apprentissage et coopération. Le déploiement de l'accordcadre confère une légitimité à ces nouvelles capacités d'action en instaurant des dispositifs de valorisation de l'apprentissage (VAE, tutorat, constitution d'un vivier de conducteurs appelés à exercer des fonctions d'encadrement...) et la possibilité de reconnaître les compétences individuelles (entretien annuel d'évaluation). Les organisations syndicales, représentant les intérêts des conducteurs de bus, développent une stratégie de résistance, matérialisée par des mouvements sociaux généralisés. L'accord finalement trouvé vise 
principalement à développer la formation sur la base d'entretiens dits "de professionnalisation». L'idée d'évaluation, et in fine de rétribution individuelle, se heurte également à une opposition syndicale. Les organisations syndicales sont pourtant de moins en moins représentatives des stratégies désormais plurielles des conducteurs. Les salariés dédiés à la conduite du tramway créent en effet leur propre syndicat pour faire valoir leur identité et ressources « de compétences». Aussi, les organisations syndicales ont parfois recours au référendum avant de prendre des positions lors d'une négociation.

\section{Cas $n^{\circ} 2$ : développer du lien social par la relation hiérarchique personnalisée en Suède}

Un second cas permet d'observer la mise en place d'un management participatif au sein d'une entreprise de transport public par bus dans une grande ville de Suède. Lorsque la firme multinationale achète l'entreprise suédoise dans le but de conquérir de nouveaux marchés, elle a connaissance des difficultés économiques de cette dernière. Le site employant 1200 conducteurs connaît une situation d'anomie suite à la déstructuration du construit social antérieur. Le passage d'une gestion publique à une gestion privée, s'inscrivant dans un mouvement national de dérégulation du marché du transport public urbain, a engendré des compressions de coûts. Par conséquent, l'âge de la retraite recule, la durée des congés payés est réduite et certaines pauses sont supprimées. L'attitude de retrait des conducteurs se manifeste alors par des taux record d'absentéisme (30\%), de turnover $(52 \%)$ et des dégradations matérielles. Le seul moyen pour les superviseurs d'avoir une prise sur les conducteurs est le recours à l'autorité. C'est dans ce contexte que les dirigeants suédois sont chargés de mettre en place le nouveau modèle de management. Ils décident de créer des petites équipes et de reconstruire une ligne managériale intermédiaire. Les managers de proximité, remplaçant les anciens superviseurs, doivent adopter une approche compréhensive vis-à-vis des conducteurs et développer des relations plus personnalisées. Leur action permet d'assouplir l'organisation du travail avec la réintroduction des pauses et une flexibilité accrue des emplois du temps. L'opposition inconditionnelle du syndicat à toute forme d'évaluation individuelle est contournée par une évaluation implicite et informelle des managers qui sont amenés à mieux 
Dossier : Pour une approche communicationnelle de l'individu au travail

connaître les conducteurs ${ }^{31}$. Cette nouvelle organisation est le fruit d'un groupe de travail composé de représentants de tous les acteurs de l'entreprise. Le point de vue des conducteurs y est intégré. Par ailleurs, certains délégués syndicaux sont amenés à occuper des positions d'encadrement ou sont affectés à la réalisation des plannings. La communication interne est réinstaurée au travers des actions managériales formalisées (comme la mise en place d'un journal interne) et surtout des discussions informelles avec les responsables d'équipe.

\section{Cas $n^{\circ} 3$ : instaurer une culture d'entreprise en Roumanie}

La filiale roumaine est une régie municipale lorsque l'entreprise multinationale emporte l'appel d'offre pour la production et la distribution des eaux de cette grande ville de Roumanie. Les premières transformations à mener sont profondes et relèvent principalement de la modernisation des infrastructures, de l'informatisation et du développement des réseaux de conduite d'eau. Ces opérations nécessitent un changement de la gestion, notamment dans les domaines comptable, commercial, logistique. La gestion du personnel est également concernée. Il s'agit tout d'abord pour l'entreprise de mener un plan social touchant $50 \%$ des effectifs. Dans un second temps, une gestion des emplois et des compétences doit être réalisée pour accompagner les changements. Il n'est plus attendu des salariés une spécialisation pointue. L'établissement d'une compétence globale est souhaité. Celle-ci est précisée dans une nouvelle cartographie des emplois, dans un souci de cohérence et de prospective. L'accent est également mis sur la formation continue, développée au sein d'un centre dédié, calqué sur la structure française de formation. La direction, représentée par des expatriés français, cherche à substituer une «logique compétence» aux anciennes pratiques de marchandage, «troc» et clientélisme qui régnaient en matière de gestion du personnel. Elle engage alors des négociations avec le syndicat, acteur incontournable qui a acquis dans le passé une place importante dans le fonctionnement de l'entreprise (systématiquement consulté pour les embauches, les mobilités et les licenciements). Si lors des premières négociations, les échanges se font sous le signe de la menace et des rapports de force, progressivement les interlocuteurs sont renouvelés et des accords de

31 Les conducteurs méritants sont gratifiés par des petits cadeaux, des possibilités d'enrichissement des tâches et un soutien lors de campagnes de recrutement pour les postes de « col blanc ». 
moyen terme sont trouvés. Tout d'abord, un fonds social, des aides financières et des dons sont octroyés. Des dispositifs visant à développer l'adhésion à l'entreprise s'instaurent progressivement : $10 \%$ des actions sont cédées à la communauté des salariés, un plan d'épargne groupe destiné à l'ensemble des salariés est déployé et des évènements regroupant les communautés professionnelles sont mis en place (comme une cérémonie de remise des médailles du travail). De telles démarches visent à développer une culture d'entreprise entendue comme "un cadre de pensée [et] un système de valeurs, enfin un système de règles » (Bernoux, 2004 : 141). Un accord de méthodes portant sur le dialogue social est signé instaurant la mise en place de commissions paritaires à différentes strates hiérarchiques. Enfin, un accord d'entreprise est signé pour trois années, fait rare à l'époque de l'ancienne régie alors habituée à négocier chaque année de nouveaux accords.

\section{La double légitimité des transferts de pratiques de management}

Un regard transversal sur ces trois cas montre que c'est au cours d'une négociation qui est "l'expression d'une conflictualité coopérative et la construction de compromis » (Lichtenberger, 2000 : 279), que les changements des règles de l'organisation trouvent une légitimité double. Celle-ci est assise, d'une part, sur les nouvelles capacités de socialisation créées par la négociation et la transformation des identités professionnelles qui en résultent, et, d'autre part, sur l'encastrement culturel des modalités d'une telle «activité communicationnelle ». Tout d'abord, la mise en place de pratiques issues du «management par les compétences » soumise à des négociations résultant de la confrontation de différentes stratégies d'acteurs, se traduit par la révision du contrat social local. De tels changements des règles de travail apparaissent légitimes à partir du moment où des transformations des formes de socialisation et des identités professionnelles s'opèrent (cf. tableau 1). Dans l'entreprise française, le développement d'une nouvelle identité par l'acquisition de compétences et la progression professionnelle prend le pas sur la socialisation par le collectif et l'identité «fusionnelle» des conducteurs. La légitimité de l'acteur syndical traditionnel est affectée dans l'«organisation apprenante» (Argyris, 1985) et conduit à l'émergence de nouveaux acteurs. Le cas suédois montre que la participation des salariés au fonctionnement de l'organisation et la restauration d'un lien social par des relations hiérarchiques personnalisées mettent fin à la situation d'anomie qui avait conduit antérieurement au retrait des salariés. Enfin, dans l'entreprise roumaine, la socialisation qui autrefois se faisait au travers de 
Dossier : Pour une approche communicationnelle de l'individu au travail

pratiques de marchandage et de l'appartenance à des réseaux informels, est désormais conditionnée par les dispositifs d'adhésion à l'entreprise. Les relations de travail transformées s'appuient sur des liens communautaires et de solidarité pour rendre les bouleversements organisationnels entrepris socialement acceptables. De plus, la légitimation des pratiques introduites se joue également dans les «modalités de la communication» (Mucchielli, 1991). Bien que le caractère «mou» du concept de culture nationale ait souvent été contesté, notamment par les auteurs de l'analyse sociétale (Maurice, Sellier, Silvestre, 1982), nos observations attestent cependant de la validité d'une interprétation culturelle des modes de relations professionnelles. La culture nationale est entendue comme «un langage, un code [et] un référentiel permettant aux acteurs de donner un sens au monde où ils vivent et à leurs actions » (D'Iribarne, 1989 : IV). Les relations professionnelles correspondent à des représentations partagées culturellement encastrées (Le Velly, 2002). En France, les multiples mouvements sociaux et oppositions syndicales à ce système nous permettent de qualifier cette régulation de «conflictuelle ». Mue par la «logique de l'honneur» (D'Iribarne, 1989), elle se manifeste selon «un jeu d'esquive qui favorise le règlement par la crise» (Reynaud, 1975 : 181). Le cas suédois montre que, sans être inscrites dans un accord formel, les nouvelles règles peuvent être créées au plus près du terrain dans la coopération et le consensus qui caractérise les relations sociales en Suède (D'Iribarne et $a l$, 2002). La logique marchande, dans le cas roumain, renvoie à la dimension manichéenne propre à la culture de ce pays (Spiridon, 2005). Dans un premier temps, les acteurs locaux acceptent des changements selon une logique de «troc » qui vise à optimiser les contreparties immédiates. C'est seulement dans un second temps que des formes de dialogue s'instaurent dans l'entreprise.

\begin{tabular}{|l|l|l|l|}
\hline & France Suè & \multicolumn{1}{|c|}{ de } & Roumanie \\
\hline $\begin{array}{l}\text { Nouvelle identité } \\
\text { professionnelle et } \\
\text { forme de socialisation }\end{array}$ & $\begin{array}{l}\text { Identité et } \\
\text { socialisation par } \\
\text { le développement } \\
\text { des compétences }\end{array}$ & $\begin{array}{l}\text { Identité par la } \\
\text { participation et } \\
\text { socialisation par } \\
\text { la relation } \\
\text { hiérarchique } \\
\text { personnalisée }\end{array}$ & $\begin{array}{l}\text { Identité et } \\
\text { socialisation } \\
\text { par } \\
\text { l'instauration } \\
\text { d'une culture } \\
\text { d'entreprise }\end{array}$ \\
\hline $\begin{array}{l}\text { Comportement } \\
\text { stratégique des acteurs }\end{array}$ & $\begin{array}{l}\text { Résistance au } \\
\text { changement }\end{array}$ & Coopération & $\begin{array}{l}\text { Optimisation } \\
\text { des } \\
\text { contreparties }\end{array}$ \\
\hline $\begin{array}{l}\text { Modalités } \\
\text { communicationnelles }\end{array}$ & Conflictuelles & Consensuelles & Marchandes \\
\hline
\end{tabular}

Tableau 1 : Processus de légitimation des transferts de pratiques de management au sein de la firme locale-globale par la négociation sociale 
La légitimité des transferts de pratiques de management...

\section{En conclusion}

La légitimité de la firme qui s'internationalise repose sur sa capacité à négocier son modèle de gestion avec les acteurs locaux. Cette « régulation conjointe» (Reynaud, 1997) lui confère un caractère «local-global»(Lorrain, 2005). Les trois exemples indiquent que, l'empreinte culturelle, qui connote les «modalités communicationnelles», ne circonscrit pas pour autant les comportements stratégiques des acteurs dans des positions immuables. Elle s'apparente à une « culture contenu» (Dupuy, 2004) qui, tout en s'exprimant dans un mode communicationnel inscrit dans un schéma national, permet aux acteurs un apprentissage en vue d'acquérir de nouvelles capacités d'action. Les identités professionnelles sont redéfinies au sens où les représentations, qui déterminent l'appartenance, évoluent.

\section{Bibliographie}

ARGYRIS C. Strategy, Change and Defensive Routines. Boston: M.A.A, Ballanger, 1985, 368 p.

BERGER S. Made in Monde. Paris : Seuil, 2006, 356 p.

BERNOUX P., BURLAT C. Mutations de l'entreprise, travail et emploi : le cas des Fusions-Acquisitions. in: TREMBLAY D-G. Flexibilité, sécurité d'emploi et flexicurité : Les enjeux et défis. Québec : Presses de l'Université du Québec, 2008, pp. 49-64.

BERNOUX P. Sociologie du changement dans les entreprises et les organisations. Paris : Seuil, 2004, 336 p.

BROCHIER D., GUITTON C., LEGAY A. et MACHADO J. Les groupes face à la réforme de la formation continue - Le cas de Veolia Environnement. in : Bref-Céreq, n ${ }^{\circ}$ 243, 2007, 4 p.

CROZIER M. et FRIEDBERG E. L'acteur et le Système. Paris : Seuil, 1977, $445 \mathrm{p}$.

DAVENPORT T-H., DE LONG D-W., BEERS, M-C. Successful knowledge management projects. in : Sloan Management Review Vol. 39, Issue 2, 1998, pp. 43-57

D'IRIBARNE P. La logique de l'honneur. Gestion des entreprises et traditions nationales. Paris : Seuil, 1989, 279 p.

D'IRIBARNE P., HENRY A., SEGAL J-P., CHEVRIER S., GLOBOKAR T. Cultures et mondialisation. - Gérer par-delà les frontières. Paris : Seuil, 2002, 357 p. 
Dossier : Pour une approche communicationnelle de l'individu au travail

DUPUY F. Sociologie du changement. Paris : Dunod, 2004, 304 p.

GUILBERT P. Apprendre dans les réorganisations : une perspective communicationnelle. in : Communication et Organisation, 2008, n ${ }^{\circ} 33$, pp. 41-48.

HABERMAS J. Théorie de l'agir communicationnel. Paris : Fayard, 1987, $480 \mathrm{p}$.

KOGUT B., ZANDER U. Knowledge of the Firm and The Evolutionary Theory of The Multinational Corporation. in : Journal of International Business Studies, Fourth Quarter 1993, pp. 625-645.

KOSTOVA T., ROTH K. Adoption of an organizational practice by subsidiaries of multinational corporations : institutional and relational effects. in : Academy of Management Journal, Vol. 45, $\mathrm{n}^{\circ}$ 1, 2002, pp. 215-233.

LE VELLY R. La notion d'encastrement: une sociologie des échanges marchands. in : Sociologie du travail, 2002, vol. $44, \mathrm{n}^{\circ} 1$, pp. 37-53.

LICHTENBERGER Y. Conflits, coopération et compromis : la négociation comme construction de réalités complexes, in: THUDEROZ C., GIRAUD-HERAUD A. La négociation sociale. Paris : CNRS Editions, 2000, pp. 265-279.

LORRAIN D. La firme locale-globale, (Lyonnaise des Eaux : 19802004). in : Sociologie du Travail, 2005, vol. 47, n³, pp. 340-361.

MAURICE M., SELLIER F., SILVESTRE J-J. Politique d'éducation et organisation industrielle en France et en Allemagne, Paris : PUF, 1982, $382 \mathrm{p}$.

MENDEZ A. L'internationalisation comme processus de création de ressources. Thèse de doctorat en sciences économiques. Aix-enProvence: Laboratoire d'Economie et de Sociologie du travail, Université d'Aix-Marseille II, 1994, 373 p.

MUCCHIELLI A. Les situations de communication. Paris : Eyrolles, 1991, $131 \mathrm{p}$.

REYNAUD J-D. Les syndicats en France. Paris: Seuil, 1975, $2^{\mathrm{e}}$ édition, Tome 2, $348 \mathrm{p}$.

REYNAUD J-D. Les règles du jeu. L'action collective et la régulation sociale. Paris : Armand Colin, 1997, 368 p. 
La légitimité des transferts de pratiques de management...

REYNAUD J-D. Le management par les compétences: un essai d'analyse, in: Sociologie du travail, vol. 43, n 1, 2001, pp. 7-31.

SAINSAULIEU R. L'identité au travail. Paris : PFNSP, 1977, 332 p.

SAINSAULIEU R. L'entreprise une affaire de société. Paris : Presses de la fondation nationale des sciences politiques, 1990, $353 \mathrm{p}$.

SPIRIDON M. Les dilemmes de l'identité aux confins de l'Europe. Le cas roumain. Paris : l'Harmattan, 2005, 188 p.

THUDEROZ C., GIRAUD-HERAUD A. La négociation sociale. Paris : CNRS Editions, 2000, 290 p.

WEBER M. Economie et Société. Tome 1. Paris : Plon, 1971, 336 p.

ZARIFIAN P. Le modèle de la compétence. Paris : Editions Liaisons, 2004, $130 \mathrm{p}$. 